\title{
Biomimetic Design Inspired Sharkskin Denticles for Growth Suppression of Biofilm
}

\author{
Mariko Miyazaki $^{{ }^{*}}$, Hiroshi Moriya ${ }^{1}$, and Akihiro Miyauchi ${ }^{2}$ \\ ${ }^{1}$ Research and Development Group, Hitachi, Ltd., 832-2 Horiguchi, Hitachinaka, Ibaraki \\ 312-0034, Japan \\ ${ }^{2}$ Tokyo Medical and Dental University, 2-3-10 Kanda-Surugadai, Chiyodaku, Tokyo 101-0062, \\ Japan \\ *mariko.miyazaki.jm@hitachi.com
}

\begin{abstract}
While the hydrodynamic function of sharkskin has been controversially hypothesized to be capable of achieving turbulent flow control and drag reduction, sharkskin-inspired "riblets" have been reported to be an effective biomimetic design (BMD). Furthermore, denticles have been found to not only improve fluid properties but also provide an antibacterial effect. We address an integrated study of developing biomimetic riblets inspired by the antibacterial properties of sharkskin denticles. The covering ratio of bacteria on samples with BMD structures was evaluated to confirm the effect of the antibacterial property. Our results indicate that as the non-uniformity of the grooves increases, the bacteria adhering to the surface is removed due to the effect of the longitudinal vortex generated on the sharkskin, and the growth of the biofilm is suppressed.
\end{abstract}

Keywords: Biomimetic riblets, Sharkskin, 3D digitizing, Antibacterial effect, Biofilm, Fluid control

\section{Introduction}

Biomimetics, that seeks sustainable solutions for practical problems by emulating nature's timetested patterns, functions and strategies, has developed remarkably in recent years [1]. For example, the adhesive nature of geckos feet was studied [2]. Geckos are known to crawl up walls, hang from ceilings, and firmly grasp slippery glasslike surfaces using their adsorptive toes. The flagella on the soles of a gecko's foot are dense, and the feet adhere by van der Waals forces working between the flagella and the surface. In addition, inspired by the fine, rough surface texture of lotus leaves, an excellent water-repellent surface was developed [3].

Sharkskin, as illustrated in Fig. 1, is covered with numerous small tooth-like elements, termed dermal denticles ("denticles" hereafter) [4,5]. The hydrodynamic function of sharkskin has investigated for more than 30 years with the hypothesis that the microstructure of denticles, i.e., the minute projections, passively controls turbulent flow and reduce drag [6-13]. Sharkskin has been observed in two dimensions by using a scanning electron microscope (SEM), as shown in Fig. 2, However, a three-dimensional (3D) detailed view of the sharkskin structure has yet to be confirmed. Therefore, for a biomimetic design (BMD) of the denticles, the "riblets," namely, the regular grooves with triangular or rectangular sections, have been studied both experimentally and computationally. Studies have confirmed that riblets are capable of reducing drag [14-21]. Actual denticles, however, in general demonstrate complicated 3D microstructures with a scale of less than $1 \mathrm{~mm}$. Along with the recent rapid development in highprecision, high-performance 3D structural observation and analysis techniques for material surfaces, it has become possible to measure the 3D micro-surface structures of living organisms such as sharks. Figure 3 shows the 3D structures of single denticles of a Galapagos shark observed by using a microfocus X-ray CT measuring system. From the 3D observation results, we proposed that denticle structures have two unique characteristics: a sloping structure with large angles of inclination (AOI) and 
grooves with distinguished heights or spacing in the water flow direction, namely non-uniform grooves [22]. We clarified that the unique structures of the denticle (sloping structure and non-uniform grooves) generate complex, 3D local turbulent flows near the denticles, for example, a rising flow and multiple longitudinal vortices aligned with the water flow direction. We also proposed a prototype biomimetic riblet design inspired by sharkskin denticles and generated a similar flow to sharkskin from a 3D digitized structure of the denticles [23]. Furthermore, we applied the BMD to the diffuser vanes of a centrifugal compressor that reduced its efficiency, and confirmed the effect of the BMD on fluid performance.

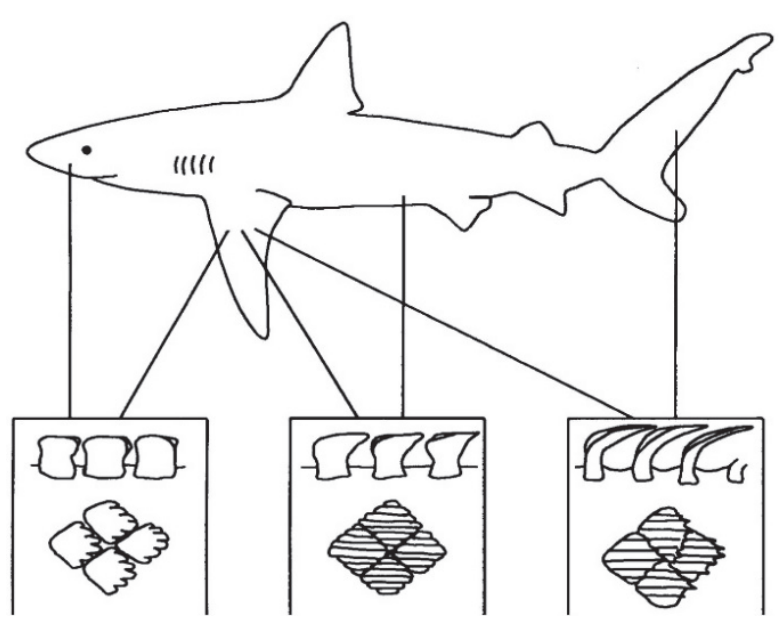

Fig. 1. Examples of denticle structures at different position in sharkskin [4].

In recent years, denticles have been found to not only improve fluid properties but also provide an antibacterial effect. An antibacterial plate (Sharklet ${ }^{\mathrm{TM}}$ ) made of silicone elastomer imitating the structure of denticle was developed, and it was reported that this plate provided an antibacterial effect in various pathogenic microorganisms (Staphylococcus aureus, Methicillin-resistant Staphylococcus aureus, Pseudomonas aeruginosa, Escherichia coli, and vancomycin resistant enterococci) [24,25]. We considered that characteristic flow fields occurring on the sharkskin, i.e., rising flow and longitudinal vortices, suppress biofilm growth as well as improve fluid properties.

In this study, we developed a biomimetic riblets design inspired by the antibacterial property of sharkskin denticles. We evaluated the effect of biofilm growth suppression using test samples of fabricated biomimetic riblets inspired by sharkskin denticles.

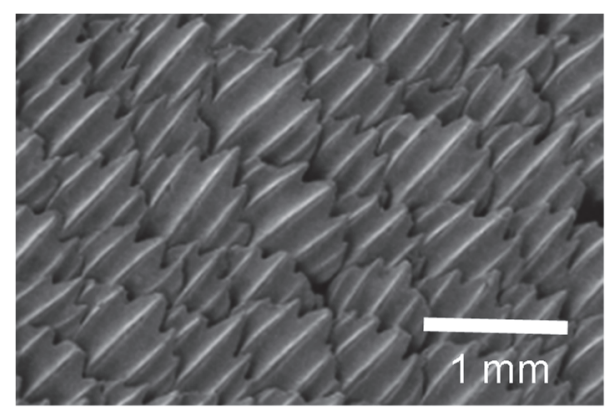

Fig. 2. Sharkskin denticles of Galapagos shark observed by SEM. (a)

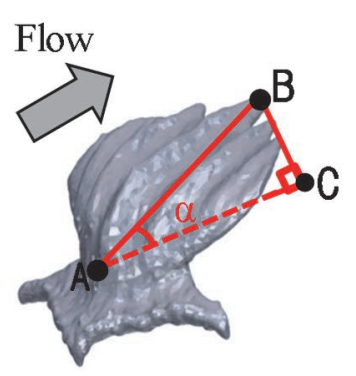

(b)

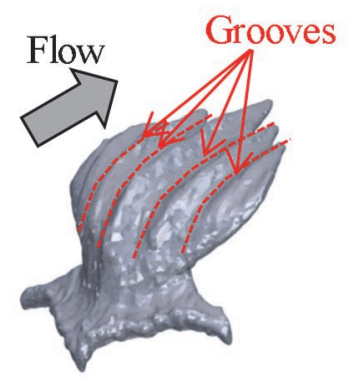

Fig. 3. 3D structures of single denticles of Galapagos shark. Structures of the denticles have two unique characteristics. (a) Sloping structure with large AOI. (b) Non-uniform grooves (with distinguished heights or spacing) in water flow direction.

\section{Experimental}

2.1. Concept of biofilm growth suppression

Biofilms clog the water intake of a power plant and filters of a seawater desalination plant, and it causes infections via a catheter in the medical field. In addition, metal corrosion due to sulfate-reducing bacteria in biofilms also causes many problems in areas of welding at plants.

Figure 4 shows the growth process of a biofilm. When a clean base material comes into contact with water, organic matter adheres immediately to the interface between water and the base material. It is interpreted as a conditioning layer for neutralizing the excess charge of the substrate and lowering the surface free energy [26]. Even if a flow field is present, the water has viscosity, so the flow velocity on the surface of the substrate is zero and a so-called retention layer is formed, which makes it easy for bacteria to adhere. The bacteria that first adhere to the organic matter layer are called pioneer bacteria, and the first adhesion mechanism is electrostatic 
attraction or physical adhesion. In the attached bacteria, extracellular polysaccharide (EPS) adheres to the substrate, and the adhesion is stabilized. After that, the pioneer bacteria begin to grow and colonies coated with polysaccharides (glycocalyx) are formed [27]. Glycocalyx takes up other types of microbial cells and secondary colonies that metabolize the excretion of primary colonies consisting of pioneer bacteria are formed in the upper layer of primary colonies (pioneer colonies). This secondary colony adheres several days after primary colony formation [28]. Afterwards, microorganisms that metabolize the excretion of the secondary colonies adhere, and a multilayered colony structure is sequentially formed into a biofilm. The completed biofilm accumulates biochemical resources inside and completes a closed space in which water, excrement, nutrients, enzymes, oxygen, and so forth, traverse inside the film. As oxygen is unlikely to enter the biofilm, anaerobic bacteria can inhabit. Antibiotics used for biofilm removal, such as antiseptic solutions, only kill colonies of the outermost layer; the internal colony group does not die because it is selfsufficient in the closed space [29]. The once-formed biofilms are difficult to remove even by using a disinfectant solution, causing problems on all substrates that come into contact with water, such as in-hospital infections and filter clogging. As such, fundamental countermeasures do not exist. To suppress the growth of biofilms in water, suppressing the formation of an organic layer on the substrate is considered to be difficult because the surface energy of the substrate in water must be lowered. In subsequent colonization of pioneer bacteria, adhesion strength is considered to be weak, especially in the initial process. The basic concept is that the formation of primary colonies is inhibited by the self-breaking of pioneer bacteria colonies on the surface of the substrate, and the growth after secondary colonies does not occur. Figure 5 shows a model of the substrate structure breaking the primary colony. This model applies a phenomenon in which microorganisms do not adhere to a sharkskin surface and breaks the primary colony by reproducing the flow field near the denticles. This model exerts its effect in the presence of flow in the water.

\subsection{Fabrication of test samples}

Samples used for the culture test of bacteria were prepared using nanoimprinting [30]. Figure 6 shows the fabrication process using nanoimprinting. A (a)

(b)

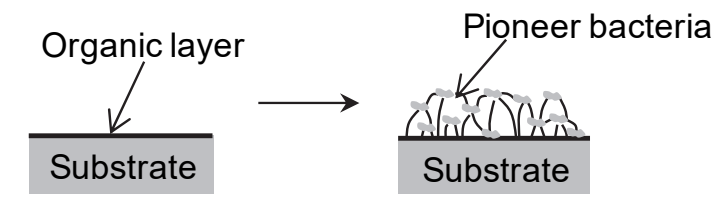

(c)

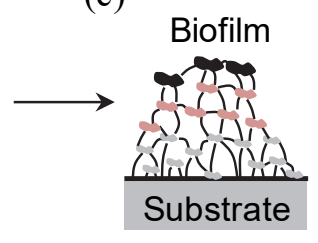

Fig. 4. Growth mechanism of bio-film in water. (a) Organic layer coating on substrate. (b) Colony formation of pioneer-bacteria. (c) Additional colony formation on pioneer-bacteria colony.

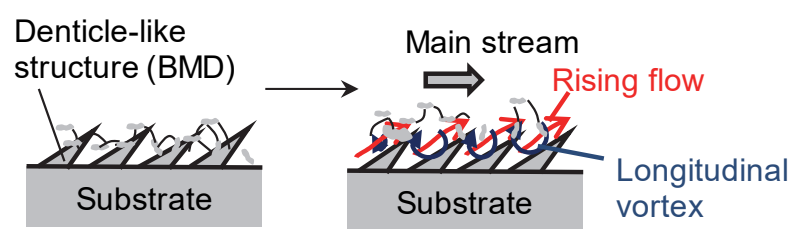

Fig. 5. Schematic diagram of disrupting biofilm growth by breaking pioneer colony.

mold with a microstructure is attached to a resin film, and heat and pressure are applied to transfer the microstructure of the mold to the resin film.

Figure 7 shows an overview of a mold in which a biomimetic-designed microstructure $\mathrm{s}$ has been fabricated. A precision cutting method was used to fabricate the mold. Figure 7 (a) shows a perspective view of the mold. The material of the mold is STAVAX, and its size is $20 \times 20 \times 10 \mathrm{~mm}$. Figure 7 (b) shows a top view of the mold. Seven types of biological deformed structure are fabricated in the mold. These structures were designed to reproduce the characteristic flow field (rising flow and longitudinal vortex) of the sharkskin as revealed in the previous study [22]. Figure 7 (c) shows a side view of the designed structure. An inclination of $27^{\circ}$ is provided. Figure 7 (d) shows a front view of the designed structure. Grooves are formed in which two triangles with different heights and widths are alternately arranged. The height $h_{1}$ and width $w_{1}$ of one triangle were fixed to 0.1 and $0.2 \mathrm{~mm}$, 
respectively. The height $h_{2}$ and width $\mathrm{w}_{2}$ of the other triangle were set to one of seven types shown in Table 1 . The ratio of the heights $\left(h_{1} / h_{2}\right)$ or widths $\left(w_{1} / w_{2}\right)$ of the two triangles represents the magnitude of the non-uniformity of the groove.
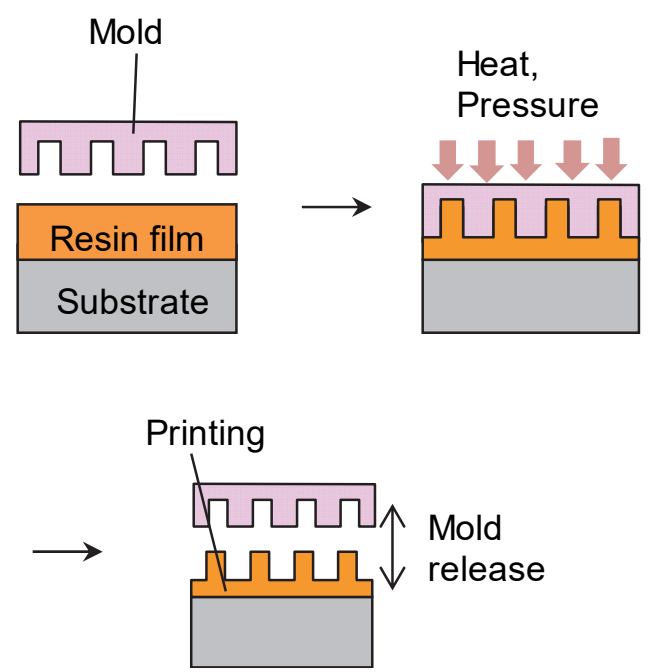

Fig. 6. Method for fabricating resin sheet by nanoimprinting. (a)

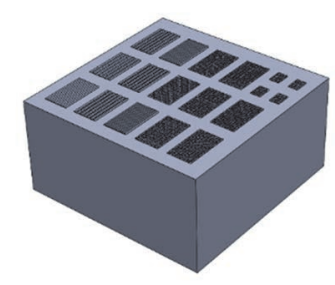

(b)

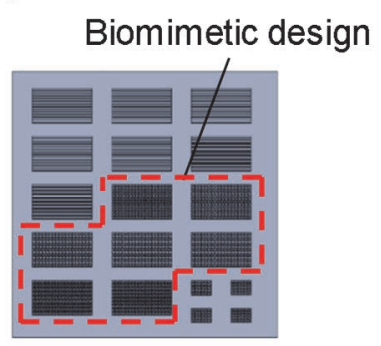

(c)

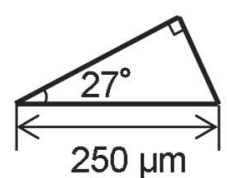

(d)

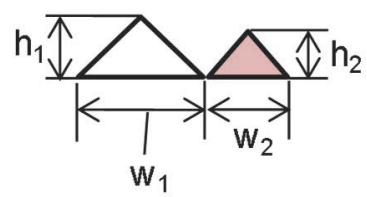

Fig. 7. Overview of mold for biomimetic design fabrication.

Table 1. Triangle height and weight values of biomimetic design.

\begin{tabular}{|c|c|c|c|c|c|c|c|}
\hline No & 1 & 2 & 3 & 4 & 5 & 6 & 7 \\
\hline $\begin{array}{c}\mathrm{h}_{2} \\
(\mathrm{~mm})\end{array}$ & 0.01 & 0.02 & 0.033 & 0.05 & 0.1 & 0.2 & 0.2 \\
\hline $\begin{array}{c}\mathrm{w}_{2} \\
(\mathrm{~mm})\end{array}$ & 0.2 & 0.2 & 0.2 & 0.2 & 0.2 & 0.066 & 0.1 \\
\hline
\end{tabular}

Procedures for fabricating a resin sheet from the mold are shown below. When transferring the structure from a metal mold directly to a resin sheet by nanoimprinting, incorrectly releasing the mold may result in the resin remaining in the mold, rendering the mold useless. Therefore, microstructures were processed on nickel by electroforming and microstructures were transferred to resin sheets by using this nickel plating foil for nanoimprint molds. As a result of examining the stability of the microstructure in advance, the ideal material of the resin was determined as cycloolefin polymer (COP). Figure 8 shows photographs of the metal molds. (a)

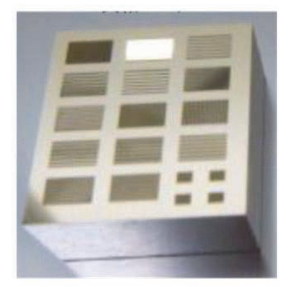

(c) (b)

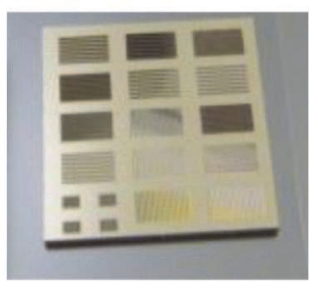

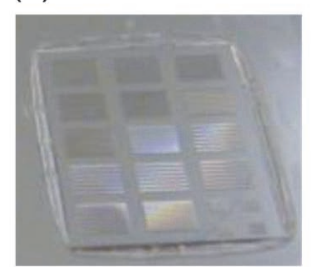

Fig. 8. Pictures of fabricated goods in processing nanoimprint. (a) Metal mold. (b) Ni plated foil. (c) COP sheet fabricated biomimetic designs.

\subsection{Method of bacterial culture}

A method for bacterial culture to verify the biofilm growth suppression effect will be described below. Figure 9 shows the design of the culture device. A resin container for the test samples was placed in a glass petri dish with an outer diameter of $90 \mathrm{~mm}$ and a height of $45 \mathrm{~mm}$. In the container, grooves were fabricated at four places, and test samples were placed therein. The top of the container was covered with a glass round plate and were secured with screws. The culture test was conducted by placing the test bacterial solution in the culture device.

The bacterial culture conditions are shown in Table 2. Staphylococcus aureus subsp. aureus NBRC 15035 was used as the test bacteria. Staphylococcus aureus is a spherical bacteria having a diameter of about $0.9 \mu \mathrm{m}$. The suspension was cultured in a trypticase soy (SCD) broth medium to obtain a bacterial culture solution in which the 
number of test bacteria was $10^{7}-10^{8} / \mathrm{mL}$. As shown in Table 2, the medium was exchanged once a day. The cultures were cultured in a state where a circulation was added using a stirrer, and samples were taken out on the fifth day from the start of the culture. The rotation speed of the stirrer was 1200 $\mathrm{rpm}$. This was determined using fluid analysis to reproduce a shark's swimming speed (about $0.5 \mathrm{~m} / \mathrm{s}$ ). Figure 10 shows a photograph of the bacteria culture using the stirrer.

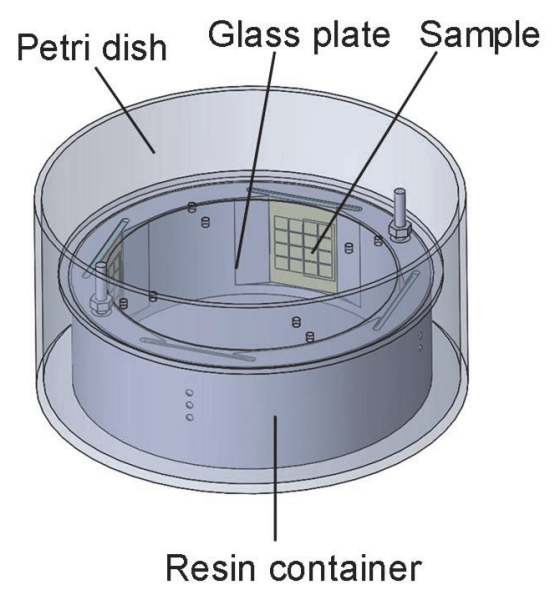

Fig. 9. Design of culture device.

Table 2. Bacterial culture conditions.

\begin{tabular}{|l|l|}
\hline $\begin{array}{l}\text { Bacteria } \\
\text { type }\end{array}$ & $\begin{array}{l}\text { Staphylococcus aureus subsp. } \\
\text { aureus NBRC } 15035\end{array}$ \\
\hline $\begin{array}{l}\text { Preparation } \\
\text { of bacteria } \\
\text { solution }\end{array}$ & $\begin{array}{l}\text { The test bacteria are cultured } \\
\text { in SCD broth medium at } \\
37 \pm 1^{\circ} \mathrm{C} \text { for } 18 \text { to } 24 \text { hours, } \\
\text { and then suspended in SCD } \\
\text { broth medium to prepare test } \\
\text { bacteria in which the number of } \\
\text { bacteria is } 10^{7} \text { to } 10^{8} / \mathrm{mL} .\end{array}$ \\
\hline $\begin{array}{l}\text { Preparation } \\
\text { of test } \\
\text { samples }\end{array}$ & $\begin{array}{l}\text { Sterilize test samples culturing } \\
\text { equipment under high pressure } \\
\text { steam sterilization (121 }\end{array}$ \\
& $\begin{array}{l}{ }^{\circ} \mathrm{C}, 15 \\
\text { minutes), and place the test } \\
\text { samples in a culture device. } \\
\text { Add } 300-350 \text { mL of the } \\
\text { bacterial solution, and incubate } \\
\text { at } 37 \pm 1{ }^{\circ} \mathrm{C} \text { by stirring at } 1200 \\
\text { rpm. }\end{array}$ \\
\hline $\begin{array}{l}\text { Test } \\
\text { operation }\end{array}$ & $\begin{array}{l}\text { Remove the test bacteria } \\
\text { solution in the sample with a } \\
\text { pipette once a day. Next, 300- } \\
350 \mathrm{~mL} \text { of purified water is } \\
\text { added, and the mixture is } \\
\text { stirred for } 1 \text { minute at } 1200 \\
\text { rpm, and the purified water is } \\
\text { removed. After repeating this } \\
\text { operation three times, add } 300 \\
-350 \mathrm{~mL} \text { of fresh SCD broth } \\
\text { medium and recultivate at } \\
37 \pm 1{ }^{\circ} \mathrm{C} .\end{array}$ \\
\hline
\end{tabular}

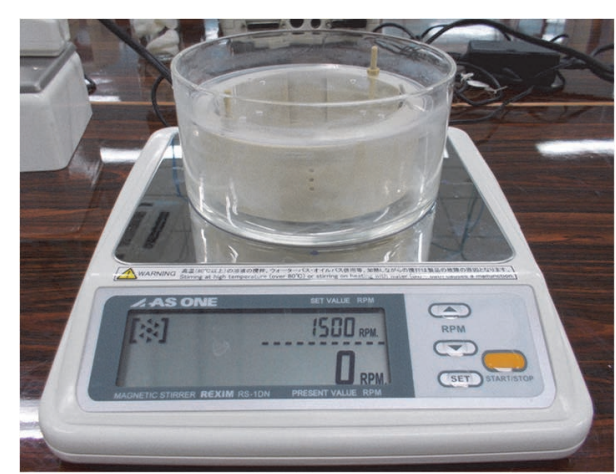

Fig. 10. Bacteria culture using stirrer.

\subsection{Method of coverage of bacteria quantification}

To quantify the bacteria covering rate of the test samples, the samples taken out after culturing for five days were gram stained and observed with an optical microscope. Gram staining is a method for dyeing bacteria with pigments. In this study, crystal violet was used as a staining solution. The test samples were immersed in a crystal violet solution for one minute and then stained by rinsing them with purified water. The covering rate of the bacteria was quantified by processing the optical microscope images obtained after dyeing. If more bacteria are attached to the test samples, more stains are observed, and the image appears darker. To quantify the covering ratio of bacteria, each pixel of the image was binarized in which black and white pixels represent areas where bacteria is and is not adhered, respectively and the covering of the bacteria is defined the following formula.

$$
\begin{aligned}
& \text { covering ratio of bacteria } \\
& =\frac{\text { Area of black area }}{\text { Total area of image }} \times 100(\%)
\end{aligned}
$$

The density was quantified for each pixel of the image, and each pixel was set to black or white with the average value as a threshold value.

\section{Results and discussion}

Figure 11 shows an optical microscopic image (200 times) of the test samples stained with crystal violet. To determine the relationship between the height non-uniformity of the groove and the adhesion state of the bacteria, we reviewed the results of $h_{1} / h_{2}=1$ (No. 5 in Table 1 ), $h_{1} / h_{2}=5$ (No. 2 in Table 1) and $h_{1} / h_{2}=10$ (No. 1 in Table 1) among the seven grooved structures fabricated into the test samples. 
(a)

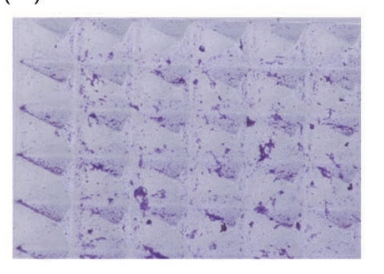

(b)

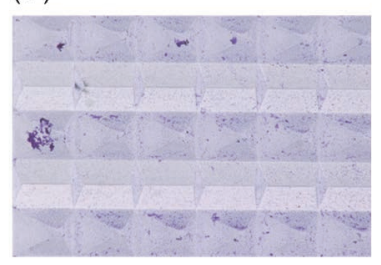

(a)

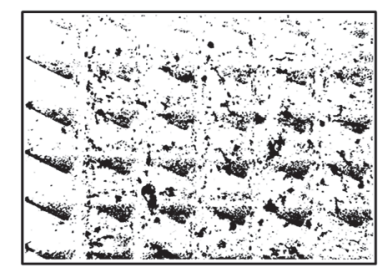

(c)
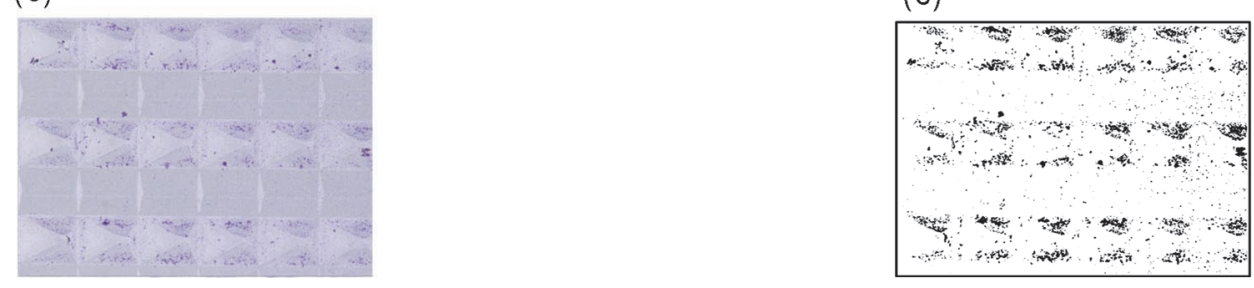

Fig. 11. Microscope image of samples stained by crystal violet $(200 \times)$. (a) $h_{1} / h_{2}=1$. (b) $h_{1} / h_{2}=5$. (c) $h_{1} / h_{2}=10$.

Areas dyed purple in the image are where the bacteria adhered in the test sample. When $h_{1} / h_{2}=1$ where the grooves are uniform, the amount of adhered bacteria was high, and as the value of $h_{1} / h_{2}$ increased where the non-uniformity of the groove increases, the amount of bacteria adhered decreases.

Figure 12 shows the processed binarized image result of the optical microscope images shown in Fig. 11. Areas in which bacteria adhered are shown in black. Figure 13 shows a graph plotting the relationship between the groove height nonuniformity $\left(h_{1} / h_{2}\right)$ and the coverage of the bacteria. When $h_{1} / h_{2}=1$ where grooves are uniform, the coverage is at the highest, and when $h_{1} / h_{2}$ increases, the coverage decreases as the groove nonuniformity increases. When $h_{1} / h_{2}=10$, the covering ratio decreased to $5 \%$; a reduction of $10 \%$ compared with that when $h_{1} / h_{2}=1$. From the images shown in Fig. 12, the bacteria adhere to most of the area in the case of $h_{1} / h_{2}=1$. On the other hand, in the case of $h_{1} / h_{2}=5$ and $h_{1} / h_{2}=10$, the bacteria adhere in a band shape. The area with much bacteria adhered has a height $h_{2}$ and is the shallow groove, and the area with few bacteria adhered has a height $h_{1}$ and is the deep groove. If the non-uniformity of the groove is large, the flow speed increases at the shallow groove due to the occurrence of the longitudinal vortex and the bacteria attached to the surface are removed by the flow. As a result, formation of the biofilm was suppressed and the coverage of the bacteria was considered to be reduced.
Fig. 12. Image processing by binarization. (a) $h_{1} / h_{2}=1$. (b) $h_{1} / h_{2}=5$. (c) $h_{1} / h_{2}=10$.

(b)
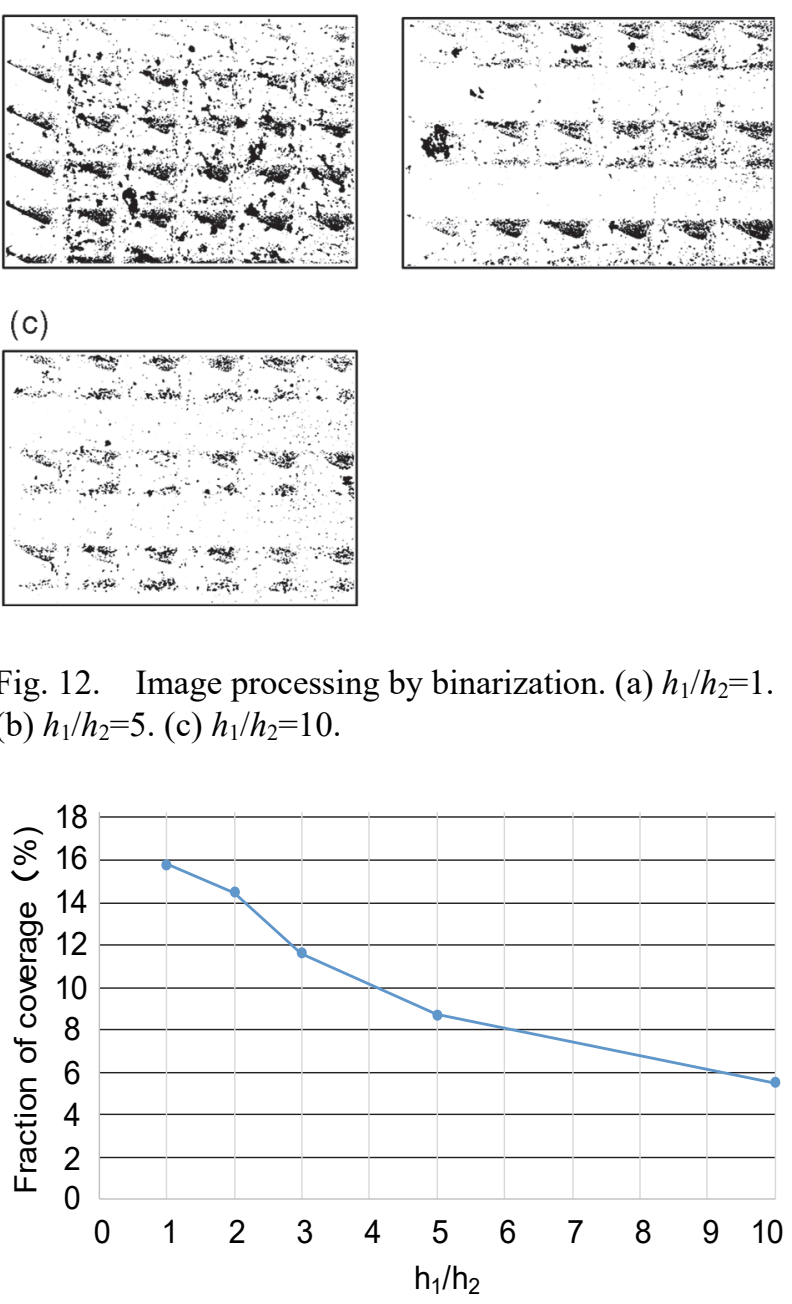

Fig. 13. Relationship between non-uniformity and fraction of coverage.

\section{Conclusion}

In this study, we focused on the antibacterial effect of sharkskin, and evaluated the effect of suppressing biofilm growth with test samples of a fabricated biomimetic riblets design inspired by sharkskin denticles. Biomimetic designed structures to generate a similar flow to sharkskin were fabricated on COP sheets by nanoimprinting, and Staphylococcus aureus was cultured on these test samples in a state where circulation was added using a stirrer. Our results indicate that as the nonuniformity of the grooves increases, the bacteria adhering to the surface is removed due to the effect of the longitudinal vortex generated on the sharkskin, and the coverage ratio of bacteria is reduced.

\section{Acknowledgement}

This work was partly supported by Advanced 
Research Program for Energy and Environmental Technologies, the New Energy and Industrial Technology Development Organization.

\section{References}

1. H. Liu, T. Nakata, G. Li, and D. Kolomenskiy, Acta Mech. Sinica, 33 (2017) 663.

2. K. Autumn, A. L. Yiching, S. T. Hsieh, W. Zesch, W. P. Chan, and T. W. Kenny, Nature, 405 (2000) 681.

3. E. P. Chan, C. Greiner, E. Arzt, and A. J. Crosby, MRS Bull., 32 (2007) 496.

4. L. Wen, J. C. Weaver, and G. V. Lauder, J. Exp. Biol., 217 (2014) 1656.

5. N. E. Kemp, Integumentary System and Teeth in Sharks, Skates, and Rays: The Biology of Elasmobranch Fishes (ed. W. C. Hamlet), Johns Hopkins University Press, (1999) 43.

6. W. Meyer and U. Seegers, J. Fish Biol., 80 (2012) 1940.

7. J. I. Castro, The Sharks of North America, Oxford University Press (2011).

8. W. E. Reif and A. Dinkelacker, Neues Jahrb. Geol. Palaontol. Abh., 164 (1982) 184.

9. W. E. Reif, Squamation and Ecology of Sharks, Frankfurt am Main: Senckenbergische Naturforschende Gesellschaft (1985).

10.D. W. Bechert, M. Bruse, W. Hage, van der Hoeven J, and G. Hoppe, J. Fluid Mech., 338 (1997) 59.

11. D. W. Bechert, M. Bruse, and W. Hage, Exp. Fluids, 28 (2000) 403.

12. A. W. Lang, P. Motta, R. Hueter, and M. Habegger, J. Mar. Technol. Soc., 45 (2011) 208.

13. M. J. Walsh, AIAA J., 72 (1980) 168.

14. M. J. Walsh, AIAA J., 21 (1983) 485.
15. P. Vukoslavcevic, J. M. Wallace, and J. I. Balint, AIAA J., 30 (1992) 1119.

16. H. Choi, P. Moin, and J. Kim J, J. Fluid Mech., 255 (1993) 503.

17. S. R. Park, AIAA J., 32 (1994) 31.

18. S. J. Lee and S. H. Lee, Exp. Fluids, 30 (2001) 153.

19. B. Dean and B. Bhushan, Phil. Trans. R. Soc., A368 (2010) 4775.

20. C. C. Buttner and U. Shulz, Smart Matter. Struct., 20 (2011) 094016.

21. Y. Luo, Y. Liu, D. Zhang, and E. Y. K. Ng, J. Mech. Med. Biol., 14 (2014) 1450029.

22. M. Miyazaki, Y. Hirai, H. Moriya, M. Shimomura, A. Miyauchi, and H. Liu, J. Bionic Eng., 15 (2018) 999.

23. M. Miyazaki, Y. Hirai, H. Moriya, M. Shimomura, A. Miyauchi, and H. Liu, $J$. Photopolym. Sci. Technol., 31 (2018) 133.

24. K. K. Chung, J. F. Schumacher, E. M. Sampson, R. A. Burne, P. J. Antonelli, and A. B. Brennan, Biointerphases, 2 (2007) 89.

25. S. T. Reddy, K. K. Chung, C. J. McDaniel, R. O. Darouiche, J. Landman, and A. B. Brennan, $J$. Endourol., 25 (2011) 1547.

26. M. W. Mittelman, Microcontamination, 3 (1985) 30.

27. D. C. Mayette, WaterReview, Water Quality Research Council, Lisle Il (1992) 1.

28. S. B. Borenstein, Microbiologically Influenced Corrosion Handbook, Industrial Press Inc., New York (1994).

29. A. Coghlan, New Sci., 15 (1996) 32.

30. K. Kuwabara and A. Miyauchi, J. Vac. Sci. Technol. B, 26 (2008) 582. 